\title{
Immune cell composition and immunological profiles of the breast cancer microenvironment represented by histologically assessed tumor- infiltrating lymphocytes and PD-L1 expression: A systematic analysis
}

\section{Toru Hanamura}

Tokai University School of Medicine https://orcid.org/0000-0002-6934-8588

\section{Shigehisa Kitano}

The cancer institute hospital of JFCR

\section{Hiroshi Kagamu}

Saitama Medical University International Medical Center: Saitama Ika Daigaku Kokusai Iryo Center

\section{Makiko Yamashita}

The cancer institute hospital of JFCR

\section{Mayako Terao}

Tokai University School of Medicine: Tokai Daigaku Igakubu Daigakuin Igaku Kenkyuka

\section{Banri Tsuda}

Tokai University School of Medicine: Tokai Daigaku Igakubu Daigakuin Igaku Kenkyuka

\section{Takuho Okamura}

Tokai University School of Medicine: Tokai Daigaku Igakubu Daigakuin Igaku Kenkyuka Nobue Kumaki

Tokai University School of Medicine: Tokai Daigaku Igakubu Daigakuin Igaku Kenkyuka

\section{Katsuto Hozumi}

Tokai University School of Medicine: Tokai Daigaku Igakubu Daigakuin Igaku Kenkyuka

\section{Naoki Harada}

Chugai Pharmaceutical Co Ltd: Chugai Pharma Taiwan Ltd

\section{Takayuki Iwamoto}

Okayama University Hospital: Okayama Daigaku Byoin

\section{Chikako Honda}

Gunma University Graduate School of Medicine School of Medicine: Gunma Daigaku Daigakuin Igakukei Kenkyuka Igakubu

\section{Sasagu Kurozumi}

International University of Health and Welfare: Kokusai Iryo Fukushi Daigaku

Naoki Niikura ( $\nabla$ niikura@is.icc.u-tokai.ac.jp ) 


\section{Research article}

Keywords: breast cancer, tumor immunity, microenvironment, tumor-infiltrating lymphocyte, programmed cell death 1 ligand 1 , flow cytometry, immune cell composition

Posted Date: July 13th, 2021

DOI: https://doi.org/10.21203/rs.3.rs-687673/v1

License: (c) (i) This work is licensed under a Creative Commons Attribution 4.0 International License. Read Full License 


\section{Abstract}

Background. A better understanding of tumor immunology can facilitate the development of new treatment strategies for various malignancies. Histologically assessed tumor-infiltrating lymphocytes (hTILs) and programmed cell death 1 ligand 1 (hPD-L1) have been established as prognostic or predictive biomarkers in certain subsets of breast cancer. However, the complexity of multiple types of immune cells is not fully understood. In this study, the immune cell fractions in breast cancer tissue and blood were evaluated to analyze their association with hTILs and hPD-L1.

Methods. In total, 45 tumor and 18 blood samples were collected from breast cancer patients. The total leukocyte counts, proportions of 11 types of immune cells in the samples, and PD-L1 expression in each fraction were evaluated using multicolor flow cytometry for both the tumor and blood samples. The hTILs and hPD-L1 were evaluated with hematoxylin and eosin staining and immunohistochemistry respectively.

Results. The immune cell composition of the blood showed a partial correlation with that of the tumor tissue; however, no significant association was found between the blood immune cell compositions and hTIL or hPD-L1 expression. A higher hTIL was associated with increased leukocyte infiltration as well as a higher proportion of $\mathrm{CD} 4^{+}$and $\mathrm{CD} 8^{+} \mathrm{T}$ cells and lower proportion of natural killer cells and natural killer $\mathrm{T}$ cells. PD-L1 was highly expressed in the monocyte/macrophage $(\mathrm{Mo} / \mathrm{M} \varphi)$, nonclassical monocyte (CD16 $\left.{ }^{+} \mathrm{Mo}\right)$, myeloid-derived suppressor cell (MDSC), dendritic cell (DC), and myeloid dendritic cell (mDC) fractions in the tumor tissues. hPD-L1 positivity was associated with increased leukocyte infiltration in the tumor tissues and PD-L1 expression in $\mathrm{Mo} / \mathrm{M} \varphi, \mathrm{CD} 16^{+} \mathrm{Mo}, \mathrm{MDSC}, \mathrm{DC}$, and $\mathrm{mDC}$ fractions.

Conclusion. There was a partial correlation in the composition of immune cells at the tumor site and that in the peripheral blood. A high proportion of hTILs reflects not only higher immune cell infiltration but also differences in the immune responses in the tumor microenvironment. Non-B-cell antigen-presenting cell fractions such as $\mathrm{Mo} / \mathrm{M} \varphi, \mathrm{CD} 16^{+} \mathrm{Mo}, \mathrm{MDSC}, \mathrm{DC}$, and $\mathrm{mDC}$ fractions are involved primarily in the PD-L1 pathway in the breast cancer microenvironments.

\section{Background}

Breast cancer is the most common malignancy in women worldwide, and it develops at a relatively younger age. Although there has been progress in multimodal treatment comprising surgery, systemic therapy, and radiation therapy, it is still difficult to cure advanced and recurrent disease [1]. The recent success in the clinical use of immune checkpoint inhibitors for multiple cancers has attracted attention in tumor immunology and emerging evidence has suggested that a better understanding of tumor immunology may lead to the development of new treatment strategies or the effective use of existing therapies [2].

Histologically assessed tumor-infiltrating lymphocytes (hTILs) can provide important prognostic information for diverse solid tumor types. They may also be of value in predicting the response to 
treatment [3]. In breast cancer, although with some controversy, hTIL has been detected more frequently in the triple-negative subtype or human epidermal growth factor receptor-2 (HER-2)-positive subtype than in the luminal subtype [3] and has also been correlated with clinicopathological factors other than subtypes $[4,5]$. With regard to prognosis, hTIL has been shown to be associated with a better prognosis for disease-free survival and overall survival in the triple-negative and HER-2 positive subtypes $[4,6]$. It has also been suggested that $\mathrm{hTIL}$ is a strong predictor of response to neoadjuvant chemotherapy in all molecular subtypes [7]. The programmed cell death 1 ligand 1 (PD-L1) was shown to be expressed in immune cells, including the T cells, B cells, macrophages, monocytes, and dendritic cells, as well as in tumor cells. PD-L1 can bind to the programmed cell death protein 1 (PD-1) expressed on activated T cells. The interactions with PD-L1 enable PD-1 signaling to counter the activation of T cells during the effector phase of the immune response [8, 9]. Histologically assessed PD-L1 expression (hPD-L1s) have been found to be more frequent in HER-2 subtypes and triple-negative subtypes than in luminal subtypes, and have also been correlated with poor prognoses, higher histological grades, and lymphatic vessel invasions [10]. Furthermore, hPD-L1 has been established clinically, as a predictor of the efficacy of atezolizumab in triple-negative advanced breast cancer [11]. Recently, different types of immune cell subsets have been evaluated primarily by immunohistochemistry $(\mathrm{IHC})$ and shown to be associated with various clinicopathological factors or prognoses. These are summarized in Table S1, and suggest their clinical significance in breast cancer [12-28].

Thus, hTIL and hPD-L1 have been established as biomarkers for breast cancer, and recent studies have shifted their attention to the various immune cell subsets that make up hTILs. However, the complexity of the multiple types of immune cells in TIL-or PD-L1 expressing cells has not yet been fully understood, because of the technical difficulties in detecting multiple types of immune cells in the tissues using conventional IHC. To address this issue, we first used multicolor flow cytometry (FCM) to assess the multiple immune cell fractions in breast cancer tissue and blood and then we analyzed the association between hTIL and hPD-L1. By performing a systematic analysis of the immune cell composition, we aimed to show that specific immunological profiles of the breast cancer microenvironment were represented histologically by hTIL and hPD-L1.

\section{Methods}

\section{Patients}

Forty-seven tumor samples from the primary site and 19 matched blood samples were obtained from the breast cancer patients regardless of the clinicopathological factors or treatment histories, except for the patients with distant metastases or a clinical complete responses to neoadjuvant chemotherapy. None of the patients in this study received irradiation or endocrine therapy prior to their surgeries. Since the amendment of the study protocol in July 2016, blood sample collection commenced, thus there were cases with no blood samples. Clinicopathological data including menopausal statuses, histories of preoperative chemotherapy, histological types, invasive tumor sizes, lymph node statuses, lymphatic involvement, vascular involvement, histological grades, estrogen receptor (ER) statuses, progesterone 
receptor (PgR) statuses, HER-2 statuses, and Ki67 labeling indexes were collected by reviewing the patients' case records. The histological grades were evaluated according to the method described by Robbins et al. [29]. The ER, PgR, and HER-2 statuses were evaluated using IHC staining. The cut-off value for ER and PgR positivity was set at $\geq 10 \%$ [30]. The HER-2 status was determined according to the ASCO CAP guideline 2013 [31]. For the reasons mentioned later, 45 tumor samples and 18 blood samples were included in the analysis. The clinicopathological characteristics of the whole cohort and the cohort with blood samples are shown in Tables S2 and, S3 respectively.

\section{TIL/PBMC preparation}

Tumor and blood samples were collected simultaneously during surgery and processed. Tumor samples were mechanically dissociated on ice with 10\% FBS-PBS, filtered using a 70-micron strainer, and washed with $10 \%$ FBS-PBS. The whole blood was collected using a collection tube containing EDTA-2Na. For both the tumor and the blood samples, the mononuclear cell components were separated using densitygradient centrifugation with a Ficoll-Paque PLUS (Cytiva Inc., Tokyo, Japan). In brief, the samples diluted with PBS were layered on Ficoll-Paque and centrifuged at $1,100 \times g$ for 20 min at $18-20^{\circ} \mathrm{C}$. Mononuclear cell components were collected, washed twice with PBS, and stored in liquid nitrogen in CELLBANKER I (Takara Bio Inc. Shiga, Japan).

\section{Flow cytometry analysis}

The cryopreserved TILs and PBMCs were thawed and washed twice with PBS containing 10\% FBS and PBS. The cell suspensions were then processed for surface staining with an antibody cocktail (Table S4) for 20 min at $4^{\circ} \mathrm{C}$. The cells were then washed with PBS containing $2 \% \mathrm{FBS}$ and resuspended in CellFix (BD Biosciences). The stained cells were detected using an LSR II Fortessa with FACS Diva software (BD Biosciences). All the analyses were performed using FlowJo software (BD Biosciences, Franklin Lakes, NJ, USA). The gating strategy is shown in Figure S1. The immune cell fractions are classified into the following fractions according to the definitions shown in Table S5: Leucocytes, Total T cells (Total T), $\mathrm{CD}^{+} \mathrm{T}$ cells $\left(\mathrm{CD} 4^{+} \mathrm{T}\right), \mathrm{CD} 8^{+} \mathrm{T}$ cells $\left(\mathrm{CD} 8^{+} \mathrm{T}\right), \mathrm{B}$ cells $(\mathrm{B})$, monocytes/macrophages $(\mathrm{Mo} / \mathrm{M} \varphi)$, nonclassical monocytes (CD16 $\left.{ }^{+} \mathrm{Mo}\right)$, myeloid-derived suppressor cells (MDSC), dendritic cells (DCs), myeloid dendritic cells (mDCs), natural killer cells (NK), minor NK cells, and natural killer T cells (NKT). Two cases with a low number of living cells (count < 1000) in the CFM analysis of the tumor tissue were excluded. A blood sample that was also identical to one of the two cases, was also excluded. As mentioned previously, 45 tumor samples and 18 blood samples were included in the analysis. The leucocyte density based on the weight of the tissue fragment and the number of viable $\mathrm{CD} 45^{+}$cells was determined using the same method as a previous study [32]. For the immune cell fraction, we determined both the percentages of each fraction in the leukocytes (\% in leukocytes) and the densities based on the weights of the tissue fragments (count/g).

\section{Histological evaluation of tumor immunity-related biomarkers}


We evaluated the histological tumor immunity-related biomarkers, as described in a previous paper [3336]. Briefly, the percentages of stromal TILs were evaluated using $4-\mu \mathrm{m}$ sections from formalin-fixed specimens stained with hematoxylin and eosin using a light microscope at $\times 200-400$ magnification. Stromal TILs were defined as mononuclear cells localized in the stromal tissue of the breast cancer. The stromal TIL count was categorized, according to the International TILs Working Group guideline, into three grades: low (0-10\%), intermediate (10-40\%), or high (40-90\%); and were scored from 0 to 2 . The denominator used to determine the TIL grade was the stromal tissue area. PD-L1 expression was assessed using IHC with rabbit monoclonal anti-PD-L1 clone SP142 (prediluted; Spring Bioscience, USA) and the Ventana Benchmark ULTRA auto staining machine. Tumors with $\geq 1 \%$ immune cells with cytoplasmic and/or membrane PD-L1 staining were determined to be PD-L1 positive. The evaluation of the stromal TIL counts and PD-L1 expression was performed by two evaluators (CH and SK). In this study, the histologically assessed TIL and PD-L1 were described as hTIL and hPD-L1, respectively, to distinguish them from the CFM-assessed data. In one case, the hTIL and hPD-L1 could not be evaluated because of insufficient tumor tissue remaining. Thus, hTIL and hPD-L1 were analyzed in the remaining 44 patients.

\section{Statistical analysis}

All the statistical analyses and graph drawings were performed using GraphPad Prism ver. 9.1.0 software. All the analyses for the correlation between the two groups were performed using the Spearman's rank correlation coefficient. For the comparison of the paired samples of the tumor and blood, the Wilcoxon test was used. For the comparison of the two unpaired groups, the Mann-Whitney $U$ test was used. A Chi-square test was used to compare clinicopathological factors between the hTILs and hPD-L1. A Fisher's exact test was used when the Chi-square test indicated a significant $P$-value $(P<0.05)$ and there were cells with a sample size of five or less.

\section{Results}

Distribution of leukocyte, and immune cell fraction determined by FCM.

Leucocyte densities based on the weights of the tissue fragments and the number of viable CD $45+$ cells was determined in the entire cohort $(n=45)$. The mean density and interquartile range of the tumorinfiltrating leukocytes were $226 \times 10^{3}$ cells $/ g$ and $80 \times 10^{3}$ to $574 \times 10^{3}$ cells/g, respectively (Fig. $1 \mathrm{a}$ ). For the immune cell fraction in the leukocytes of the tumor tissues (TILs), the main population consisted of $\mathrm{CD}^{+} \mathrm{T}, \mathrm{CD} 4^{+} \mathrm{T}, \mathrm{Mo} / \mathrm{M} \varphi$, and $\mathrm{B}$ (Fig. $1 \mathrm{~b}$ and d). There was a similar trend in the leukocyte composition in blood (PBMC), showing that the main population consisted of $\mathrm{CD} 8^{+} \mathrm{T}$ and $\mathrm{CD} 4^{+} \mathrm{T}$ cells (Fig. $1 \mathrm{c}$ and d).

Immune cell composition of blood is partly correlated with that of tumor tissue but the abundance ratio of each fraction itself is different between the two.

For cases with matched samples of blood and tumor tissue, a correlation analysis was performed for the percentages of each of the immune cell fractions (Fig. 2a-I). The proportions of DC, mDC, NK, and minor 
NK in tumor tissue showed a positive correlation with those of blood (Fig. $2 \mathrm{~h}-\mathrm{k}$ ). The percentages of each immune cell fraction of tumor tissue (TIL) and that of blood (PBMC) were compared, the same cases (Fig. 3a-I). The proportions of DC, mDC, and minor NK were significantly higher in the tumor tissues compared with the proportions in blood (Fig. 3h, i, and k). On the other hand, the proportions of $\mathrm{CD} 4{ }^{+} \mathrm{T}$ and NK cells were significantly lower in tumor tissues compared with the proportions in blood (Fig. $3 \mathrm{~b}$ and j). Although we investigated the relationship between the immune cell composition in blood and hTIL or hPD-L1, there were no significant associations found between them (Figure S2 and S3). Similarly, no significant associations were found between hPD-L1 and the FCM assessed PD-L1 positive ratios in the immune cell fraction of blood (Figure S4).

Histologically assessed TIL (hTIL) was associated with not only the degree of leukocyte infiltration in tumor tissue but also its composition.

For cases with tumor tissue samples, a correlation analysis was performed for the hTIL scores and the leukocyte densities (count/g) in the tumor tissues, showing a strong positive correlation with each other (Fig. 4a). Furthermore, the hTIL scores showed a positive correlation with the densities (count/g) of almost all of the immune cell fractions in the tumor tissues, except for NK (Figure S5). A Correlation analysis was also performed for the hTIL scores and percentages of each immune cell fraction in the tumor tissues. We found that there were positive correlations between the hTIL scores and the percentages of total $\mathrm{T}, \mathrm{CD} 4^{+} \mathrm{T}$, and $\mathrm{CD} 8^{+} \mathrm{T}$ (Fig. $4 \mathrm{~b}-\mathrm{d}$ ), but negative correlations between the $\mathrm{hTIL}$ scores and NK and NKT (Fig. 4k, m), showing that hTIL was associated with not only the degree of leukocyte infiltration in tumor tissue but also its composition.

Histologically assessed PD-L1 (hPD-L1) expressions are associated with leukocyte infiltration in tumor tissue and reflect PD-L1 expression in certain immune cell fraction.

The percentages of the PD-L1 positive cells in each immune cell fraction was determined for the tumor tissues (TILs) and blood (PBMC) using FCM. Percentages of the PD-L1 positive cells of each immune cell fraction in the tumor tissues are shown in Fig. 5a, indicating that PD-L1 is preferentially expressed in nonB-cell antigen-presenting cell fractions such as $\mathrm{Mo} / \mathrm{M} \varphi, \mathrm{CD} 16^{+} \mathrm{Mo}, \mathrm{MDSC}, \mathrm{DC}$, and $\mathrm{mDC}$. For cases with matched samples of blood and tumor tissue, the percentages of the PD-L1 positive cells in each immune cell fraction were compared between the tumor tissues and blood. The PD-L1 positive ratios were significantly higher in the tumor tissues than in blood for all lineages except for the lymphoid fractions (Fig. $5 b-I$ ). Next, we investigated the relationship between hPD-L1 and the immunological profiles of the tumor tissues. Comparisons of the leukocyte densities (count/g) in the tumor tissues between the hPDL1-negative and -positive cases showed that hPD-L1 positivity was associated with increased leukocyte infiltrations in the tumor tissues (Fig. 6a). Similarly, hPD-L1 showed a positive correlation with the densities (count/g) of almost all the immune cell fractions in the tumor tissues, except B and NK (Figure S6). With regard to the immune cell composition in the tumor tissues, although hPD-L1 positivity was associated with a lower percentage of NK and NKT, it was not correlated with the percentages of other lineages (Fig. 6b-m). For tumor tissue samples, PD-L1 positive ratios in each immune cell fraction were 
compared between the hPD-L1 positive and-negative cases. We found that hPD-L1 positivity showed a positive association with the PD-L1 positive ratio in some of the immune cell fractions, including $\mathrm{Mo} / \mathrm{M} \varphi$, $\mathrm{CD}_{16}{ }^{+} \mathrm{Mo}, \mathrm{DC}$, and $\mathrm{mDC}$, but not with the other lineages (Fig. 7a-k). These data suggest that the histologically assessed PD-L1 expression reflects leukocyte infiltration in the tumor tissues and PD-L1 expression in certain immune cell fractions.

\section{Discussion}

In this study, we evaluated multiple immune cell fractions in both breast cancer tissues and matched blood samples, then observed that there was a partial correlation in the composition of immune cells at the tumor site and in the peripheral blood. In the comprehensive analysis of the association between each immune cell lineage fraction with hTIL and hPD-L1, we found for the first time that clinically diagnosed breast cancers, especially in cases with higher hTIL or positive hPD-L1 were already in a state in which innate immune responses were poorly involved or had escaped, and acquired immune responses were active. We also found that Non-B-cell antigen-presenting cell fractions were involved primarily in the PDL1 pathway in breast cancer microenvironments.

To the best of our knowledge, most of the analyses of the immune cell compositions of breast cancer tissues using a multicolor FCM had 10 colors or less [32,37], and that there were only two studies with more than 11 colors $[38,39]$. Although the reactivities of the labeled antibodies were not always the same, and a direct comparison was not possible, there was a similar distribution of the leukocyte infiltrations in the tumor tissue in our study and a previous study with a distribution median of $218 \mathrm{CD} 45^{+} \mathrm{TIL} / \mathrm{mg}$ of tumor tissue (interquartile range: $85-445 \mathrm{CD} 45^{+} \mathrm{TIL} / \mathrm{mg}$ ) [32]. Although there are very few reports of systematic examinations of leukocyte compositions in breast cancer tissue, some studies have reported a ratio of total T being $86 \%$ (mean) [32] or 75\% (median) [38] of the leukocytes (CD45 ${ }^{+}$cells) in breast cancer tissues, suggesting that T cells accounted for the majority of TILs [40]. In our study, the proportion of total T cells in the leukocytes in the tumors was $57.3 \%$ (mean), which was slightly lower than the proportion in previous reports probably due to the difference in the antibody used and the gating strategy. But consistent with the fact that T cells account for the majority of leukocytes in the tumor tissues. In three previous studies, the proportions of $\mathrm{CD} 19^{+} \mathrm{B}$ cells in CD $45^{+}$TIL were found to be $8 \%$ (mean), $4.58 \%$ (median), and approximately $10 \%$ (mean), respectively [32,37,38], which was similar to the results of our study. With regard to other lineages, the findings of a previous study showed that $C D 14^{+} / \mathrm{CD} 40^{+} / \mathrm{CD} 163^{+}$ M2 macrophages were $0.06 \%$ (median), CD $11 b^{+} /$CD $15^{+} /$HLA-DR- MDSCs were $1.19 \%$ (median), and CD56 ${ }^{+}$NK was $2.33 \%$ (median) [38]. However, the number of studies were small, and the definitions of each lineage did not match those in our study, therefore valid comparisons could not be made. No comparable reports were found for the remaining lineages.

This is the first study to show an association between the immune cell composition of blood and that of breast cancer tissues. The immune cell composition of blood showed a partial correlation with the tumor tissues (Fig. 2) and the percentages of the immune cell fractions showed some differences between the 
tumor tissues and blood (Fig. 3). While this suggested that the composition of tumor-infiltrating immune cells may be estimated using blood samples, there were significant differences between them. Each also has a different function or clinical significance.

Although there was a significant difference between the subtypes, hTIL was shown to correlate with some clinicopathological factors including subtypes [3-5], prognoses, and responses to chemotherapy $[4,6,7]$ in breast cancer. In our study, higher hTIL scores were associated with high-grade tumors, ER-negativity, higher Ki67 positive ratios, and hPD-L1 positivity (Table S6). Many studies have also reported that ERpositive breast cancer is the least immune-infiltrated subtype, which was consistent with our results $[2,5]$. However, there are some controversies with regard to other clinicopathological factors, and results differ from study to study $[2,4,5,41]$. To date, no studies have systematically assessed the relationship between $\mathrm{hTIL}$ and the immune cell fraction using FCM. In this study, we demonstrated that hTIL was associated with not only the degree of leukocyte infiltration in the tumor tissues but also the composition (Fig. 4). We found that while there were positive correlations between the hTIL scores and percentages of total $\mathrm{T}, \mathrm{CD} 4^{+} \mathrm{T}$, and $\mathrm{CD} 8^{+} \mathrm{T}$ (Fig. $\left.4 \mathrm{~b}-\mathrm{d}\right)$, there were negative correlations between the $\mathrm{hTIL}$ scores and NK and NKT (Fig. 4k, m). Therefore, we speculate that a higher hTIL not only reflects the amount of immune cell infiltration, but also reflects the state in which acquired immunity is activated, relative to innate immunity in clinically diagnosed breast cancers. Furthermore, they may suggest that there is still room for the development of cancer immunotherapy to promote acquired immune responses in breast cancers other than triple negative breast cancer.

As mentioned previously, PD-L1 plays a significant role in the immune tolerance mechanisms that suppress T-cell activation [8,9], and its expression is suggested to reflect ongoing (or active) immune responses or in addition to immunosuppression via the PD-1/PD-L1 pathway [39]. The hPD-L1 was shown to correlate with some clinicopathological factors, including subtypes [10]. It is also a clinically approved predictive marker for atezolizumab in triple-negative advanced breast cancer [11]. In the present study, hPD-L1 positivity was associated with ER-negative diseases and higher hTIL scores but no significant association with the other factors, probably due to the small cohort size (Table S7). Although PD-L1 expression in multiple types of immune cells or tumor cells has been reported [8,9], there is no consensus as to which immune cell fraction is responsible for the substantial function of the PD-L1 pathway in breast cancer. The findings of only one report that evaluated the PD-L1 expression in CD4 ${ }^{+} \mathrm{T}$ and $C D 8^{+}$T, B showed that the overall proportion of the PD-L1 positive TILs was very low and could only be detected in a small number of tumors [39]. In the present study, we found that a substantial proportion of $\mathrm{PD}-\mathrm{L} 1$ positive immune cells were non-B-cell antigen-presenting cell fractions such as $\mathrm{Mo} / \mathrm{M} \varphi, \mathrm{CD} 16^{+}$ Mo, MDSC, DC, and $\mathrm{mDC}$ fractions (Fig. 5a), and that the PD-L1 positive ratios were significantly higher in tumor tissues than in blood (Fig. 5b-I), suggesting that these fractions were involved primarily in the PDL1 pathway in breast cancer tissue. In addition, we found that hPD-L1 positive tumors exhibited increased leukocyte infiltration in tumor tissues (Fig. 6a), and that the hPD-L1 reflected PD-L1 expression in Mo/M $\varphi$, CD16 ${ }^{+}$Mo, DC, and mDCs (Fig. 7a-k). These results suggested that hPD-L1 expression indicates the activation status of the immune tolerance mechanism that occurs in non-B-cell antigen-presenting cells in 
response to an increased immune cell infiltration, mainly effector cells which secrets IFN-gamma to induce PD-L1 expression on various cells, into the breast cancer microenvironment.

The FCM analysis findings will be useful in the exploration of new immune-related factors in breast cancer. Briefly, by evaluating the expression of candidate proteins related to tumor immunity by $\mathrm{IHC}$ and analyzing them together with these data, function of candidate proteins may be verified. Currently, we are focusing on some candidate proteins as immuno-regulatory factors in breast cancer and further analyzing FCM data of this study to validate their immunological functions.

This study had several limitations. A relatively small number of patients were enrolled in this study. In addition, in a pilot study, it was found empirically that the number of cells required for FCM was not sufficient in cases of ER-positive breast cancer, especially in cases with lower Ki67s. In addition, cases of small tumor sizes and post-NAC with pathological CRs were excluded due to technical problems in the collection of the tumor tissues. Therefore, it should be noted that there was an inevitable bias in the enrollment of the cases; it differed from the general breast cancer cohort in terms of larger invasive tumor sizes, more ER-negative cases, and higher Ki67 cases (Table S2). Although, as mentioned above, the significance of the TIL is suggested to be different between subtypes, a subgroup analysis could not be performed because of the small sample size. It is recommended that in future studies, more samples be collected and more detailed analyses be performed.

\section{Conclusions}

In this study, there was a partial correlation in the composition of immune cells at the tumor site and that in the peripheral blood. A comprehensive analysis of the immune cell fractions revealed the immunological profiles of breast cancer tissue represented by hTIL or hPD-L1. Our findings indicated that hTIL not only reflected the amount of immune cell infiltration, but also the state in which acquired immunity is activated relative to innate immunity. Non-B-cell antigen-presenting cell fractions such as $\mathrm{Mo} / \mathrm{M} \varphi, \mathrm{CD} 16^{+} \mathrm{Mo}, \mathrm{MDSC}, \mathrm{DC}$, and mDCs were involved primarily in the PD-L1 pathway in breast cancer microenvironments. In addition, hPD-L1 reflects PD-L1 expression in these immune cell fractions. Our findings provide a basic understanding of the immune response in the breast cancer microenvironment and contributes to the further development of tumor immunology.

\section{Abbreviations}

Histologically assessed tumor infiltrating lymphocytes: hTIL

Human epidermal growth factor receptor-2: HER-2

Programmed cell death 1 ligand 1: PD-L1

Programmed cell death 1: PD-1 
Histologically assessed PD-L1 expression: hPD-L1

Immunohistochemistry: IHC

Flow cytometry: FCM

Estrogen receptor: ER

Progesterone receptor: $\mathrm{PgR}$

Total T cell: Total T

$\mathrm{CD}^{+}{ }^{+} \mathrm{T}$ cell: $\mathrm{CD} 4^{+} \mathrm{T}$

$\mathrm{CD}^{+} \mathrm{T}$ cell: $\mathrm{CD}^{+} \mathrm{T}$

B cell: $B$

Monocyte/Macrophage: Mo/M $\varphi$

Nonclassical monocyte: $\mathrm{CD} 16^{+} \mathrm{Mo}$

Myeloid-derived suppressor cells: MDSC

Dendritic cell: DC

Myeloid dendritic cell: mDC

Natural killer cell: NK

Natural killer T cell: NKT

\section{Declarations}

\section{Ethics approval and consent to participate}

This study was conducted at Tokai University Hospital with the approval of the Ethics Committee (Accepted project No. 16R-279), and the patients were enrolled from May 2015 to April 2019. Written informed consent was obtained from all the patients.

\section{Consent for publication}

Not applicable. 
Request for additional details of the data used in the manuscript can be directed to the corresponding author.

\section{Competing interests}

TH, ShK, HK, TO, NK, SaK, and NN received honorarium from Chugai Pharmaceutical Co., Ltd. ShK has an advisory role in Chugai Pharmaceutical Co., Ltd. HK has an advisory role in ImmuniT Research Inc. ShK, HK and NN received research funding from Chugai Pharmaceutical Co., Ltd. NH received salary from Chugai Pharmaceutical Co., Ltd. The remaining authors declare no competing interests.

\section{Funding}

This study was supported by a Grant-in-Aid for Scientific Research from the Ministry of Education, Culture, Sports, Science and Technology, Japan (Project No.18K16266; to NN), and the 2020 Tokai University School of Medicine Research Aid (to TH). This work was supported by the Chugai Pharmaceutical Co., Ltd.

\section{Authors' contributions}

TH analyzed and interpreted the data and wrote the manuscript. MT, BT, TO, and NN collected the samples. The following authors performed the experiment: HK (FCM), SK and MY (processing of the MCF data), NK, CH, and SK (the histological assessment). KH processed the samples for the FCM and supervised the writing of the manuscript. NN, TI, SK, and NH developed the concept, designed the study, and supervised the writing of the manuscript. All the authors read and approved the final version of this manuscript.

\section{Acknowledgments}

We would like to thank Editage (www.editage.com) for English language editing.

\section{References}

1. Harbeck N, Gnant M. Breast cancer. Lancet. 2017;389(10074):1134-50.

2. Dieci MV, Griguolo G, Miglietta F, Guarneri V. The immune system and hormone-receptor positive breast cancer: Is it really a dead end? Cancer treatment reviews. 2016;46:9-19.

3. Hendry S, Salgado R, Gevaert T, Russell PA, John T, Thapa B, Christie M, van de Vijver K, Estrada MV, Gonzalez-Ericsson PI, et al. Assessing Tumor-infiltrating Lymphocytes in Solid Tumors: A Practical Review for Pathologists and Proposal for a Standardized Method From the International Immunooncology Biomarkers Working Group: Part 1: Assessing the Host Immune Response, TILs in Invasive Breast Carcinoma and Ductal Carcinoma In Situ, Metastatic Tumor Deposits and Areas for Further Research. Adv Anat Pathol. 2017;24(5):235-51. 
4. Yu X, Zhang Z, Wang Z, Wu P, Qiu F, Huang J. Prognostic and predictive value of tumor-infiltrating lymphocytes in breast cancer: a systematic review and meta-analysis. Clin Transl Oncol. 2016;18(5):497-506.

5. Solinas C, Carbognin L, De Silva P, Criscitiello C, Lambertini M. Tumor-infiltrating lymphocytes in breast cancer according to tumor subtype: Current state of the art. Breast. 2017;35:142-50.

6. Mao Y, Qu Q, Chen X, Huang O, Wu J, Shen K. The Prognostic Value of Tumor-Infiltrating Lymphocytes in Breast Cancer: A Systematic Review and Meta-Analysis. PloS one. 2016;11(4):e0152500.

7. Denkert C, von Minckwitz G, Darb-Esfahani S, Ingold Heppner B, Klauschen F, Furlanetto J, Pfitzner B, Huober J, Schmitt W, Blohmer JU, et al. Abstract S1-09: Evaluation of tumor-infiltrating lymphocytes (TILs) as predictive and prognostic biomarker in different subtypes of breast cancer treated with neoadjuvant therapy - A metaanalysis of 3771 patients. Cancer research. 2017;77(4 Supplement):109.

8. Pardoll DM. The blockade of immune checkpoints in cancer immunotherapy. Nature reviews Cancer. 2012;12(4):252-64.

9. Cimino-Mathews A, Thompson E, Taube JM, Ye X, Lu Y, Meeker A, Xu H, Sharma R, Lecksell K, Cornish TC, et al. PD-L1 (B7-H1) expression and the immune tumor microenvironment in primary and metastatic breast carcinomas. Human pathology. 2016;47(1):52-63.

10. Li X, Li M, Lian Z, Zhu H, Kong L, Wang P, Yu J. Prognostic Role of Programmed Death Ligand-1 Expression in Breast Cancer: A Systematic Review and Meta-Analysis. Target Oncol. 2016;11(6):753-61.

11. Schmid P, Adams S, Rugo HS, Schneeweiss A, Barrios CH, Iwata H, Dieras V, Hegg R, Im SA, Shaw Wright G, et al. Atezolizumab and Nab-Paclitaxel in Advanced Triple-Negative Breast Cancer. N Engl J Med. 2018;379(22):2108-21.

12. Mahmoud SM, Paish EC, Powe DG, Macmillan RD, Grainge MJ, Lee AH, Ellis IO, Green AR. Tumorinfiltrating CD8 + lymphocytes predict clinical outcome in breast cancer. Journal of clinical oncology: official journal of the American Society of Clinical Oncology. 2011;29(15):1949-55.

13. Ali HR, Provenzano E, Dawson SJ, Blows FM, Liu B, Shah M, Earl HM, Poole CJ, Hiller L, Dunn JA, et al. Association between CD8 + T-cell infiltration and breast cancer survival in 12439 patients. Ann Oncol. 2014;25(8):1536-43.

14. Gao G, Wang Z, Qu X, Zhang Z: Prognostic value of tumor-infiltrating lymphocytes in patients with triple-negative breast cancer. a systematic review and meta-analysis. BMC cancer 2020, 20(1):179.

15. Schnellhardt S, Erber R, Büttner-Herold M, Rosahl MC, Ott OJ, Strnad V, Beckmann MW, King L, Hartmann A, Fietkau R, et al: Tumour-Infiltrating Inflammatory Cells in Early Breast Cancer: An Underrated Prognostic and Predictive Factor? International journal of molecular sciences 2020, 21(21).

16. Burugu S, Asleh-Aburaya K, Nielsen TO. Immune infiltrates in the breast cancer microenvironment: detection, characterization and clinical implication. Breast cancer (Tokyo Japan). 2017;24(1):3-15. 
17. Kim ST, Jeong H, Woo OH, Seo JH, Kim A, Lee ES, Shin SW, Kim YH, Kim JS, Park KH. Tumorinfiltrating lymphocytes, tumor characteristics, and recurrence in patients with early breast cancer. Am J Clin Oncol. 2013;36(3):224-31.

18. Liu S, Foulkes WD, Leung S, Gao D, Lau S, Kos Z, Nielsen TO. Prognostic significance of FOXP3 + tumor-infiltrating lymphocytes in breast cancer depends on estrogen receptor and human epidermal growth factor receptor-2 expression status and concurrent cytotoxic T-cell infiltration. Breast cancer research: BCR. 2014;16(5):432.

19. Mahmoud SM, Paish EC, Powe DG, Macmillan RD, Lee AH, Ellis IO, Green AR. An evaluation of the clinical significance of FOXP3 + infiltrating cells in human breast cancer. Breast cancer research treatment. 2011;127(1):99-108.

20. Gu-Trantien C, Loi S, Garaud S, Equeter C, Libin M, de Wind A, Ravoet M, Le Buanec H, Sibille C, Manfouo-Foutsop G, et al. CD4ân ${ }^{\circ}$ follicular helper T cell infiltration predicts breast cancer survival. J Clin Investig. 2013;123(7):2873-92.

21. Mahmoud SM, Lee AH, Paish EC, Macmillan RD, Ellis IO, Green AR. The prognostic significance of B lymphocytes in invasive carcinoma of the breast. Breast cancer research treatment. 2012;132(2):545-53.

22. Qiu SQ, Waaijer SJH, Zwager MC, de Vries EGE, van der Vegt B, Schröder CP. Tumor-associated macrophages in breast cancer: Innocent bystander or important player? Cancer treatment reviews. 2018;70:178-89.

23. Zhang QW, Liu L, Gong CY, Shi HS, Zeng YH, Wang XZ, Zhao YW, Wei YQ. Prognostic significance of tumor-associated macrophages in solid tumor: a meta-analysis of the literature. PloS one. 2012;7(12):e50946.

24. Li F, Zhao Y, Wei L, Li S, Liu J. Tumor-infiltrating Treg, MDSC, and IDO expression associated with outcomes of neoadjuvant chemotherapy of breast cancer. Cancer Biol Ther. 2018;19(8):695-705.

25. de la Cruz-Merino L, Barco-Sánchez A, Henao Carrasco F, Nogales Fernández E, Vallejo Benítez A, Brugal Molina J, Martínez Peinado A, Grueso López A, Ruiz Borrego M, Codes Manuel de Villena M et al: New insights into the role of the immune microenvironment in breast carcinoma. Clin Dev Immunol 2013, 2013:785317.

26. Lee H, Lee HJ, Song IH, Bang WS, Heo SH, Gong G, Park IA: CD11c-Positive Dendritic Cells in Triplenegative Breast Cancer. In Vivo 2018, 32(6):1561-1569.

27. Tsuge $T$, Yamakawa $M$, Tsukamoto $M$. Infiltrating dendritic/Langerhans cells in primary breast cancer. Breast cancer research treatment. 2000;59(2):141-52.

28. Rathore AS, Goel MM, Makker A, Kumar S, Srivastava AN. Is the tumor infiltrating natural killer cell (NK-TILs) count in infiltrating ductal carcinoma of breast prognostically significant? Asian Pacific journal of cancer prevention: APJCP. 2014;15(8):3757-61.

29. Robbins P, Pinder S, de Klerk N, Dawkins H, Harvey J, Sterrett G, Ellis I, Elston C. Histological grading of breast carcinomas: a study of interobserver agreement. Human pathology. 1995;26(8):873-9. 
30. Allred DC, Harvey JM, Berardo M, Clark GM. Prognostic and predictive factors in breast cancer by immunohistochemical analysis. Modern pathology: an official journal of the United States Canadian Academy of Pathology Inc. 1998;11(2):155-68.

31. Wolff AC, Hammond ME, Hicks DG, Dowsett M, McShane LM, Allison KH, Allred DC, Bartlett JM, Bilous M, Fitzgibbons P, et al. Recommendations for human epidermal growth factor receptor 2 testing in breast cancer: American Society of Clinical Oncology/College of American Pathologists clinical practice guideline update. Journal of clinical oncology: official journal of the American Society of Clinical Oncology. 2013;31(31):3997-4013.

32. Buisseret L, Garaud S, de Wind A, Van den Eynden G, Boisson A, Solinas C, Gu-Trantien C, Naveaux C, Lodewyckx JN, Duvillier $\mathrm{H}$, et al. Tumor-infiltrating lymphocyte composition, organization and PD-1/ PD-L1 expression are linked in breast cancer. Oncoimmunology. 2017;6(1):e1257452.

33. Kurozumi S, Inoue K, Matsumoto H, Fujii T, Horiguchi J, Oyama T, Kurosumi M, Shirabe K. Clinicopathological values of PD-L1 expression in HER2-positive breast cancer. Scientific reports. 2019;9(1):16662.

34. Kurozumi S, Kaira K, Matsumoto H, Hirakata T, Yokobori T, Inoue K, Horiguchi J, Katayama A, Koshi $H$, Shimizu A, et al. $\beta$ (2)-Adrenergic receptor expression is associated with biomarkers of tumor immunity and predicts poor prognosis in estrogen receptor-negative breast cancer. Breast cancer research treatment. 2019;177(3):603-10.

35. Kurozumi S, Matsumoto H, Kurosumi M, Inoue K, Fujii T, Horiguchi J, Shirabe K, Oyama T, Kuwano H. Prognostic significance of tumour-infiltrating lymphocytes for oestrogen receptor-negative breast cancer without lymph node metastasis. Oncol Lett. 2019;17(3):2647-56.

36. Salgado R, Denkert C, Demaria S, Sirtaine N, Klauschen F, Pruneri G, Wienert S, Van den Eynden G, Baehner FL, Penault-Llorca F, et al: The evaluation of tumor-infiltrating lymphocytes (TILs) in breast cancer: recommendations by an International TILs Working Group 2014. Annals of oncology: official journal of the European Society for Medical Oncology 2015, 26(2):259-271.

37. Garaud S, Buisseret L, Solinas C, Gu-Trantien C, de Wind A, Van den Eynden G, Naveaux C, Lodewyckx JN, Boisson A, Duvillier $\mathrm{H}$, et al: Tumor infiltrating B-cells signal functional humoral immune responses in breast cancer. $J C l$ Insight 2019, 5(18).

38. Duechler M, Peczek L, Zuk K, Zalesna I, Jeziorski A, Czyz M. The heterogeneous immune microenvironment in breast cancer is affected by hypoxia-related genes. Immunobiology. 2014;219(2):158-65.

39. Solinas C, Garaud S, De Silva P, Boisson A, Van den Eynden G, de Wind A, Risso P, Rodrigues Vitória J, Richard F, Migliori E, et al. Immune Checkpoint Molecules on Tumor-Infiltrating Lymphocytes and Their Association with Tertiary Lymphoid Structures in Human Breast Cancer. Frontiers in immunology. 2017;8:1412.

40. Whitford P, Mallon EA, George WD, Campbell AM. Flow cytometric analysis of tumour infiltrating lymphocytes in breast cancer. British journal of cancer. 1990;62(6):971-5. 
41. Stanton SE, Disis ML. Clinical significance of tumor-infiltrating lymphocytes in breast cancer. J Immunother Cancer. 2016;4:59.

\section{Figures}

\section{Figure 1}

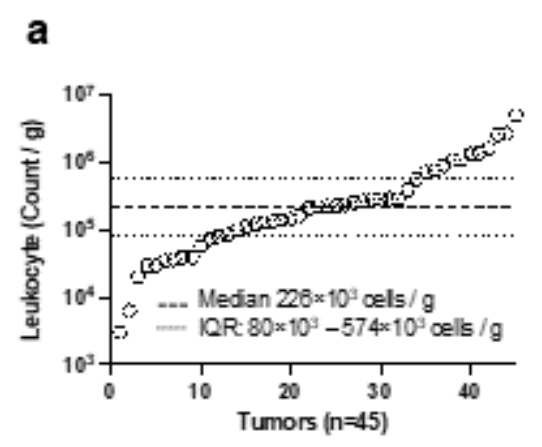

d
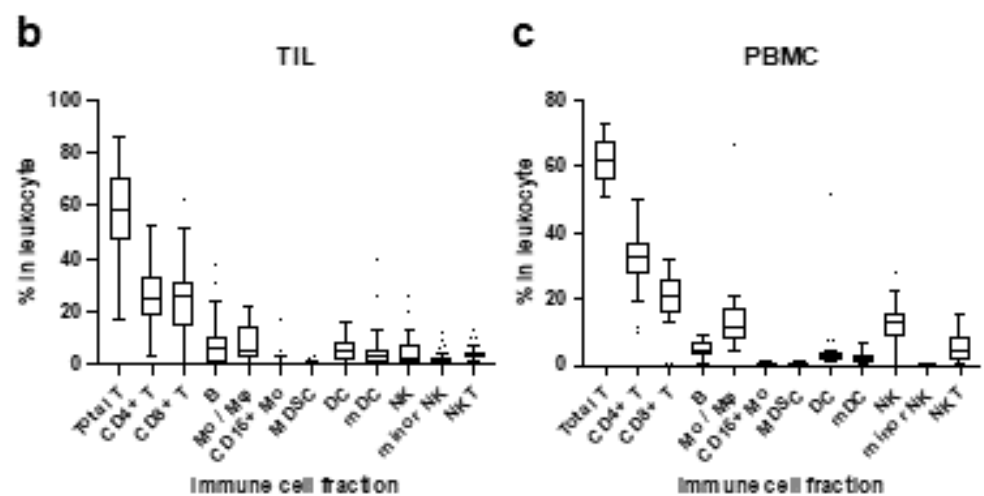

\begin{tabular}{|c|c|c|c|c|}
\hline \multicolumn{5}{|c|}{$\begin{array}{l}\text { Summary of statisticalvalues } \\
\text { (percentage in leukocyte; TIL) }\end{array}$} \\
\hline & Mean & Median & $\mathrm{SD}$ & $I Q R$ \\
\hline Total T & 57.3 & 58.9 & 17.5 & 20.8 \\
\hline $\mathrm{CD} 4+\mathrm{T}$ & 26.5 & 25.4 & 11.8 & 13.5 \\
\hline $\mathrm{CD} 8+\mathrm{T}$ & 24.3 & 25.7 & 13.5 & 16.4 \\
\hline B & 9.1 & 5.9 & 11.1 & 9.8 \\
\hline Mo / M $\varphi$ & 11.3 & 5.8 & 13.5 & 10.4 \\
\hline CD16+ Mo & 1.4 & 0.7 & 2.6 & 1.1 \\
\hline MDSC & 0.7 & 0.3 & 1.1 & 0.6 \\
\hline $\mathrm{DC}$ & 7.2 & 5.2 & 8.0 & 6.5 \\
\hline $\mathrm{mDC}$ & 5.0 & 3.4 & 7.0 & 4.7 \\
\hline NK & 4.8 & 2.4 & 5.5 & 6.1 \\
\hline minor NK & 3.5 & 1.3 & 7.9 & 1.6 \\
\hline NKT & 4.2 & 36 & 25 & 25 \\
\hline
\end{tabular}

\begin{tabular}{|c|c|c|c|c|}
\hline $\begin{array}{l}\text { Summary } \\
\text { (percentag }\end{array}$ & $\begin{array}{l}\text { of stat } \\
\text { ge in le }\end{array}$ & calvalu & & \\
\hline & Mean & Median & SD & IQR \\
\hline Total T & 58.0 & 62.2 & 16.4 & 11.3 \\
\hline $\mathrm{CD} 4+\mathrm{T}$ & 31.3 & 33.0 & 9.7 & 7.7 \\
\hline $\mathrm{CD} 8+\mathrm{T}$ & 20.1 & 21.3 & 9.0 & 8.1 \\
\hline B & 5.3 & 4.3 & 3.3 & 3.3 \\
\hline Mo / M $\varphi$ & 15.0 & 11.6 & 13.8 & 8.6 \\
\hline CD16+ Mo & 0.8 & 0.7 & 0.9 & 0.6 \\
\hline MDSC & 0.5 & 0.4 & 0.4 & 0.4 \\
\hline DC & 5.8 & 2.9 & 11.6 & 2.1 \\
\hline $\mathrm{mDC}$ & 5.0 & 2.4 & 10.5 & 2.1 \\
\hline NK & 12.5 & 13.2 & 7.2 & 6.8 \\
\hline minor NK & 0.2 & 0.2 & 0.2 & 0.2 \\
\hline NKT & 5.9 & 4.2 & 5.7 & 5.7 \\
\hline
\end{tabular}

\section{Figure 1}

Summary of the distribution of the leukocytes and immune cell fractions determined using FCM. Tumor and blood samples were assessed using multicolor FCM. The leucocyte and 11 types of immune cell fraction in the samples were determined according to the definitions in the Materials and Methods section. (a) The distribution of leukocyte density (count/g) determined using MCF for 45 breast tumors is shown graphically with the medians, 25th percentile and 75th percentile. (b, c) Percentages of each immune cell fractions are shown for the tumors $(n=45)$ and blood samples $(n=18)$ using Tukey box plots. (d) Statistical values are summarized for the percentages of each immune cell fractions in the tumor tissues $(n=45)$. 
Figure 2

Summary of statistical values

\begin{tabular}{lcc}
\hline Linage & Spearman \\
\hline Total T & 0.096 & P-value \\
CD4+ T & 0.269 & 0.705 \\
CD8+ T & 0.465 & 0.052 \\
B & 0.234 & 0.350 \\
Mo / M $~$ & 0.224 & 0.372 \\
CD16+ Mo & 0.058 & 0.820 \\
MDSC & -0.04 & 0.874 \\
DC & 0.682 & $0.002^{*}$ \\
mDC & 0.672 & $0.002^{*}$ \\
NK & 0.752 & $<.001^{*}$ \\
minor NK & 0.678 & $0.002^{*}$ \\
NKT & 0.131 & 0.604 \\
\hline Statisticaly
\end{tabular}

"Statistically significant $P$-value
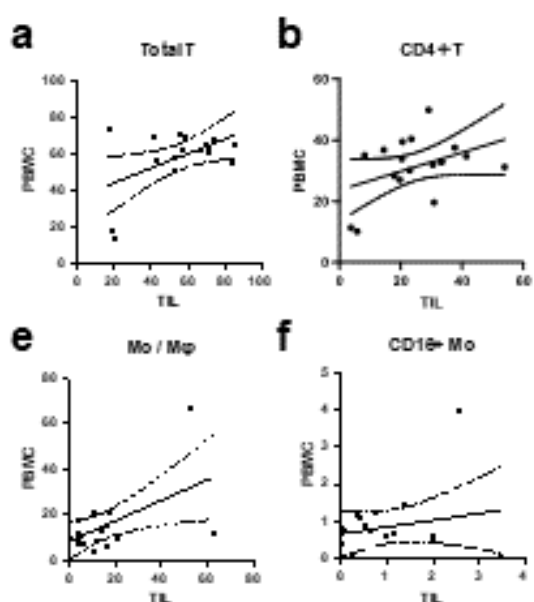

f
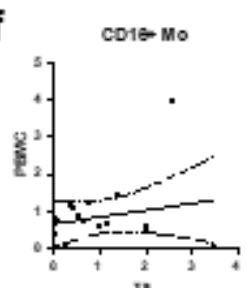

g

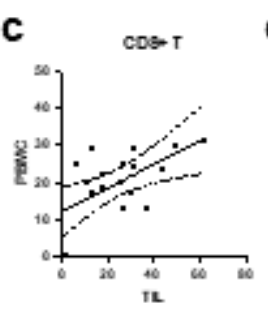

d $\quad$ s

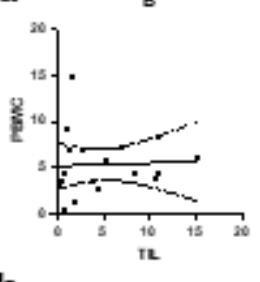

mosc

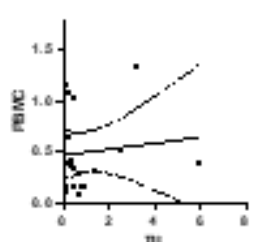

h $\quad$ c

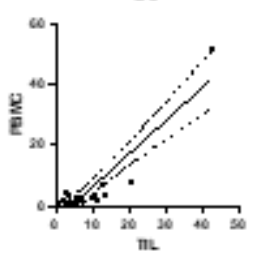

Nex
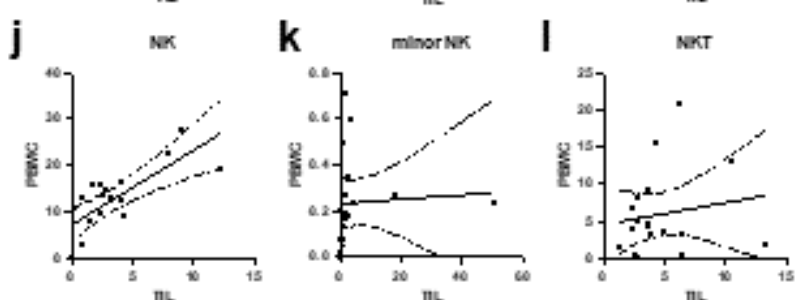

\section{Figure 2}

Immune cell composition of blood is partly correlated with that of tumor tissue. (a-l) For cases with matched samples of blood and tumor tissues, a correlation analysis was performed for the percentage of each immune cell fraction. The $\mathrm{X}$ - and $\mathrm{Y}$-axes show the percentage of immune cell fractions for the tumor tissue (TIL) and blood (PBMC), respectively. The lines in the graph indicate the regression line with a 95\% confidence band. The relationship between these two values were analyzed using the Spearman correlation. Values of $\mathrm{P}<0.05$ were considered statistically significant. Statistical values are summarized in the table on the left side of the figure. 
Figure 3
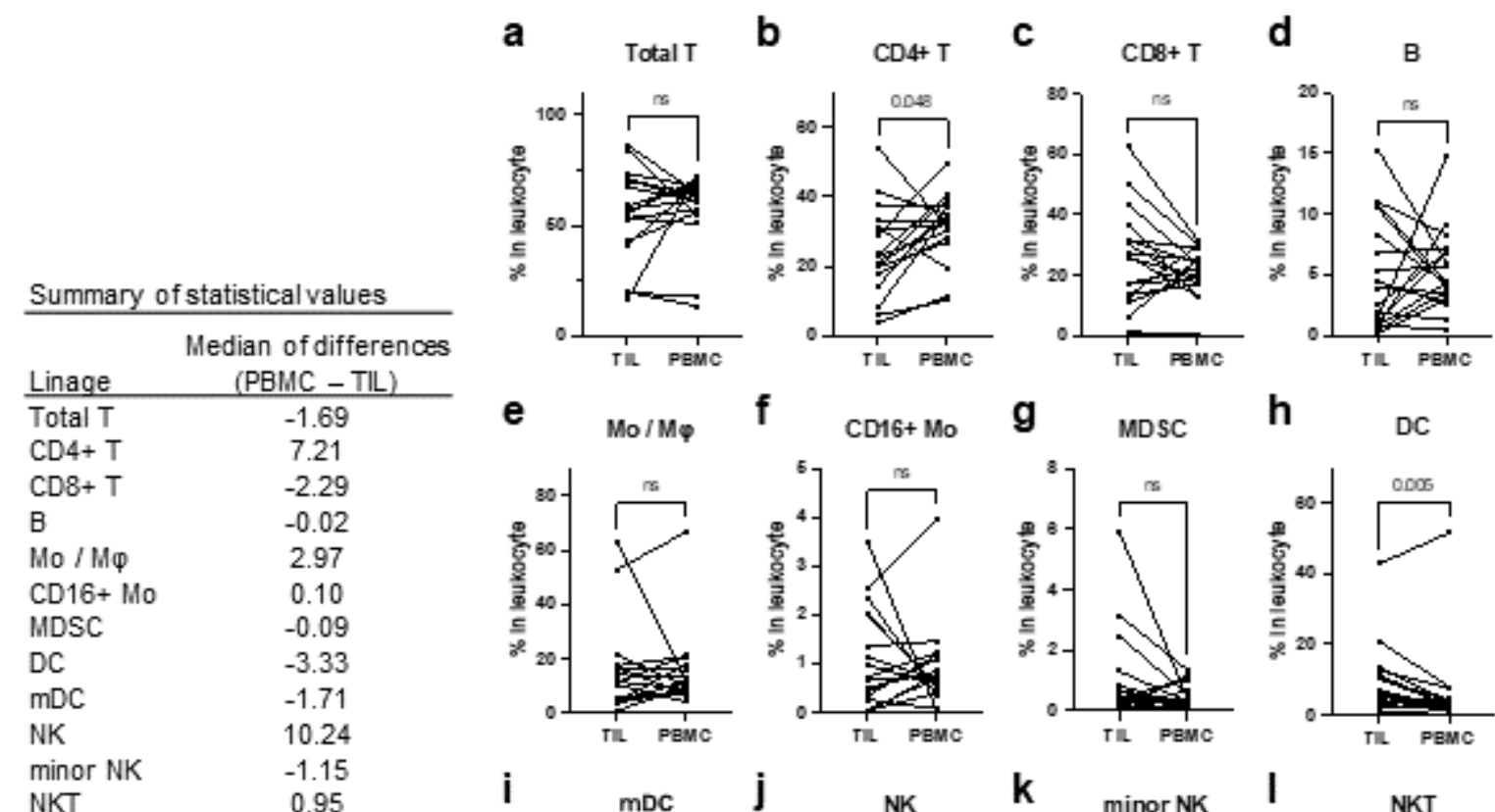

h DC
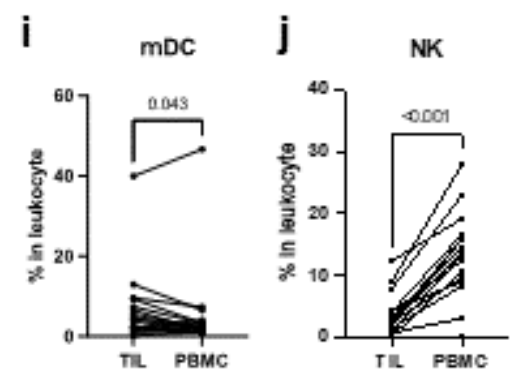

k minor NK I
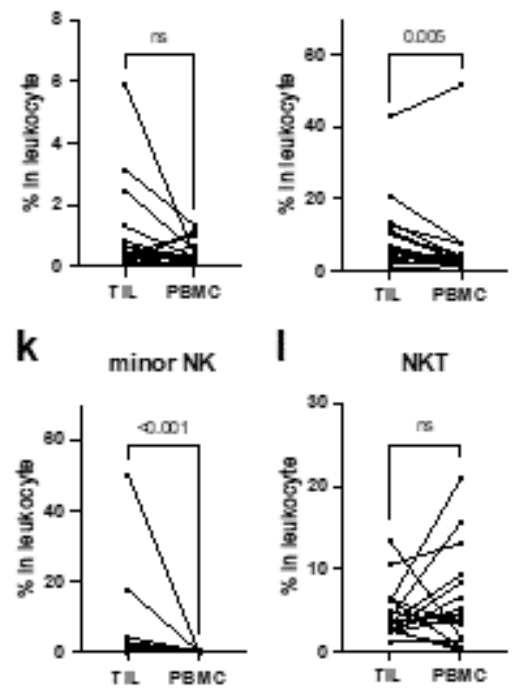

\section{Figure 3}

The percentage of immune cell fractions is partly different between tumor tissue and blood. (a-l) For 18 cases with matched samples of blood and tumor tissues, the percentages of each immune cell fraction of the tumor tissues (TIL) and of blood (PBMC) were compared using the Wilcoxon test. Values of $P$ $<0.05$ were considered statistically significant. The actual P-values are shown in the graphs. The medians of the differences are summarized in the table on the left side of the figure. 
Figure 4
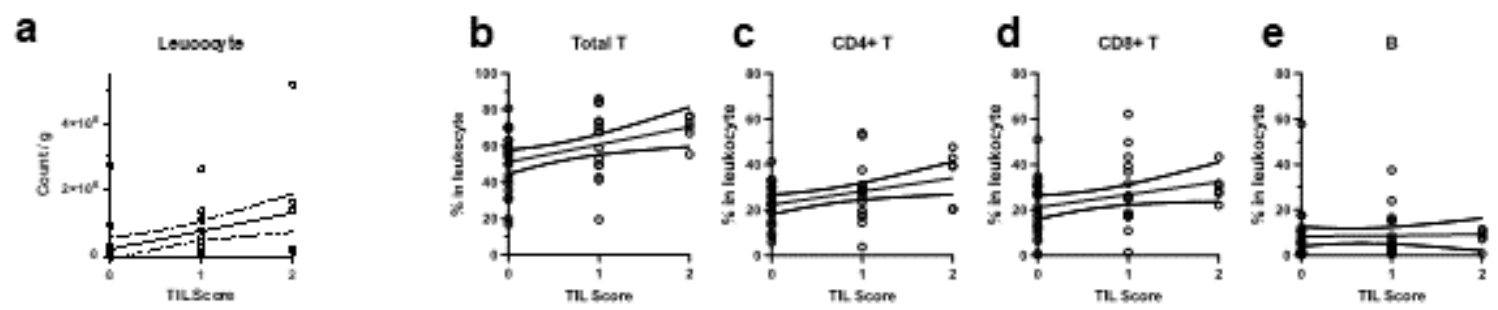

\begin{tabular}{|c|c|c|}
\hline \multicolumn{3}{|c|}{ Summary of statisticalvalues } \\
\hline & Spearman $r$ & P-value \\
\hline Leucocyte & 0.547 & $<.001^{*}$ \\
\hline Total T & 0.405 & $0.006^{*}$ \\
\hline $\mathrm{CD} 4+\mathrm{T}$ & 0.316 & $0.037^{*}$ \\
\hline $\mathrm{CD} 8+\mathrm{T}$ & 0.323 & $0.032^{*}$ \\
\hline B & 0.163 & 0.289 \\
\hline Mo / $M \varphi$ & -0.286 & 0.060 \\
\hline CD16+Mo & -0.036 & 0.816 \\
\hline MDSC & -0.080 & 0.605 \\
\hline DC & -0.207 & 0.178 \\
\hline $\mathrm{mDC}$ & -0.290 & 0.056 \\
\hline NK & -0.495 & $0.001^{*}$ \\
\hline minor NK & 0.022 & 0.886 \\
\hline NKT & -0.472 & $0.001^{*}$ \\
\hline
\end{tabular}
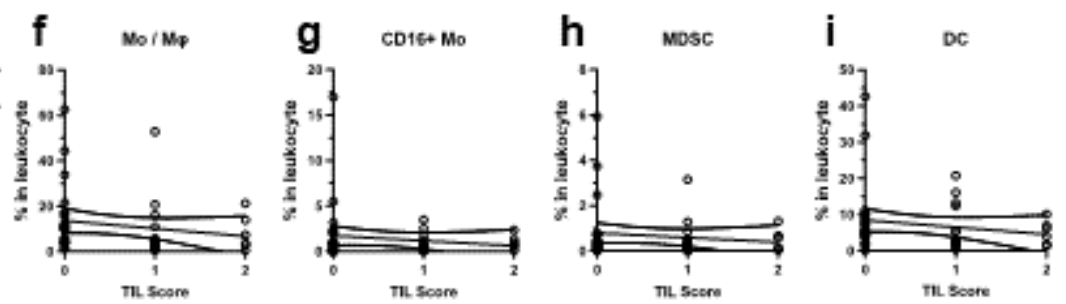

*Statistically significant $P$-value
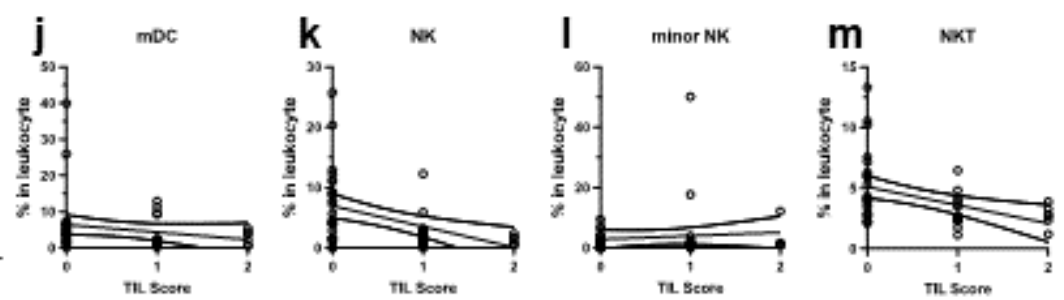

\section{Figure 4}

hTIL was associated with not only the degree of leukocyte infiltration in tumor tissue but also its composition. For cases with tumor tissue samples, a correlation analysis was performed for (a) hTIL scores and leukocyte densities (count/g) in tumor tissues, and $(b-m)$ The $h$ TIL scores and percentages of each immune cell fraction in the tumor tissues. The X-and Y-axis show the hTIL scores and (a) leukocyte densities (count/g) or $(b-m)$ the percentages of the immune cell fractions in the tumor tissue. The lines in the graph indicate the regression line with a 95\% confidence band. The relationship between the two values were analyzed using a Spearman correlation. Values of $\mathrm{P}<0.05$ were considered statistically significant. Statistical values are summarized in the table on the left side of the figure. 
Figure 5

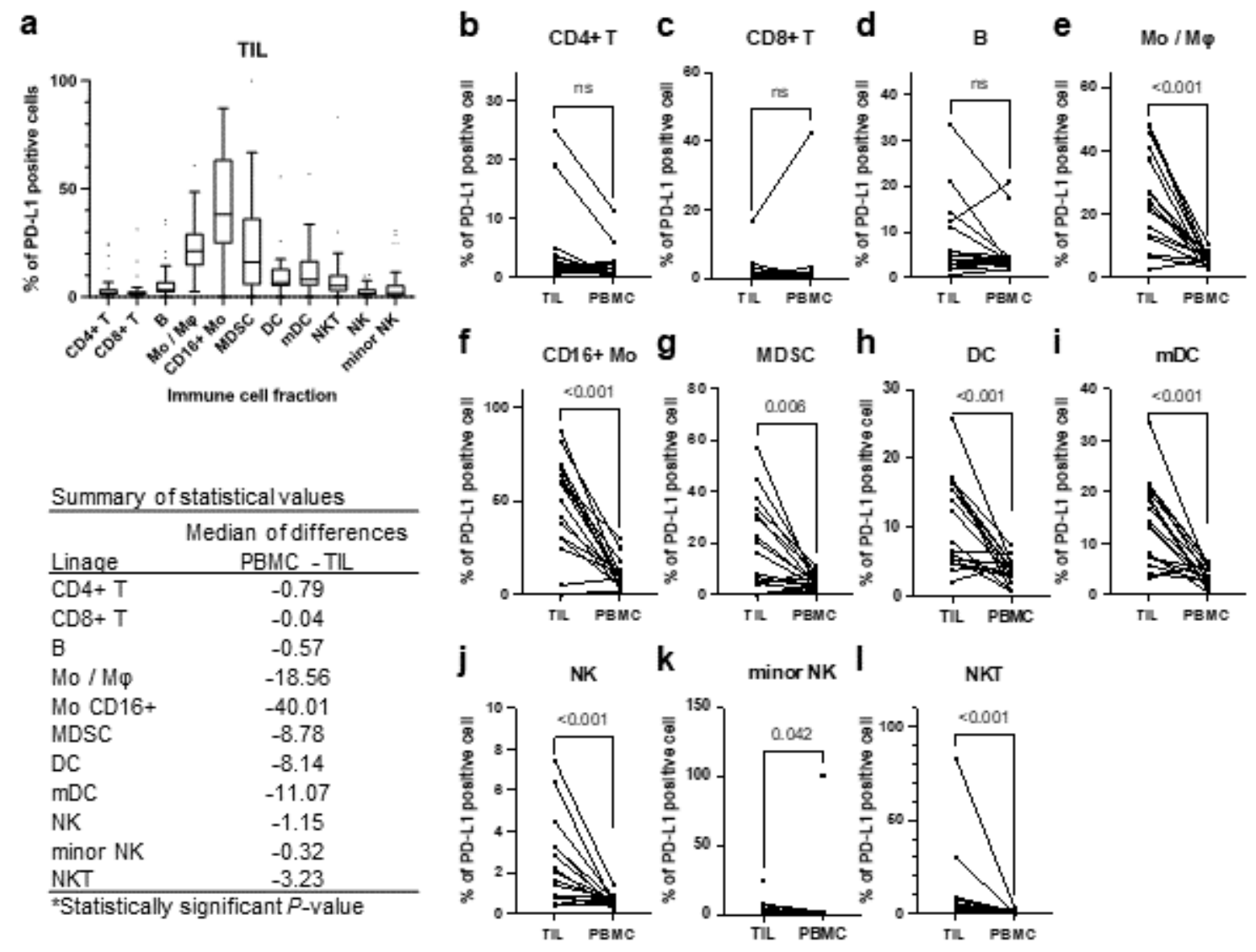

Figure 5

PD-L1 is preferentially expressed in part of the immune cell fraction, and higher in tumor infiltrating leukocytes than in blood. The percentages of PD-L1 positive cells of each immune cell fractions were determined for the tumor tissues (TIL) and blood (PBMC). (a) For the tumor tissue samples, the percentages of PD-L1 positive cells in each immune cell fraction are shown using Tukey box plots. (b-l) For the cases with matched samples of blood and tumor tissue, the percentages of PD-L1 positive cells of each immune cell fractions were compared for the tumor tissue (TIL) and that of blood (PBMC) using the Wilcoxon test. Values of $\mathrm{P}<0.05$ were considered statistically significant. The actual $\mathrm{P}$-values are shown in the graphs. The median of the differences are summarized in the table on the left side of the figure. 
Figure 6

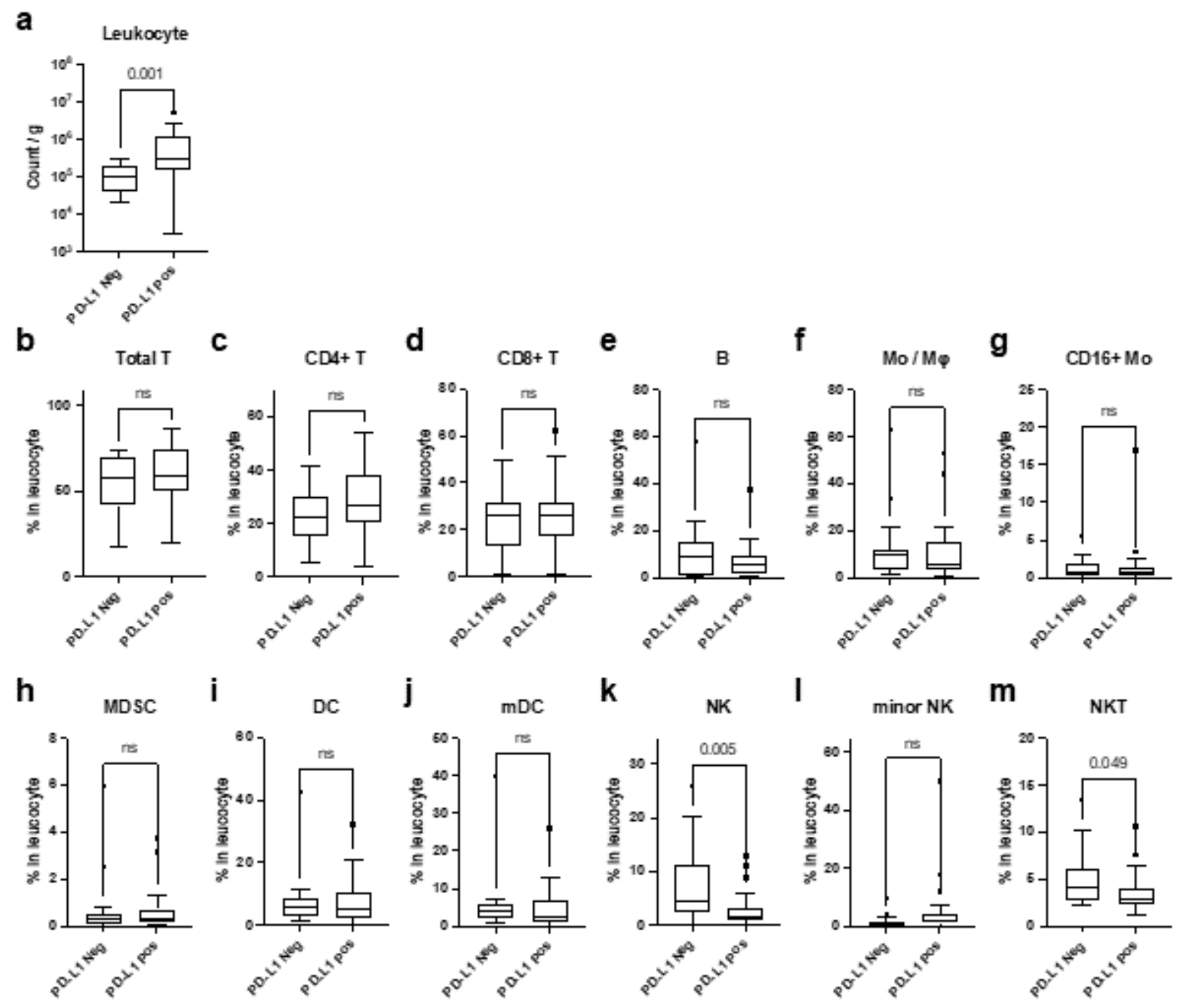

Figure 6

hPD-L1 positivity was associated with increased leukocyte infiltrations in the tumor tissue and partly with the immune cell composition. For cases with tumor tissue samples, (a) leukocyte densities (count/g) and $(b-m)$ the percentages of the immune cell fractions in the tumor tissues were compared between the hPD-L1-negative cases and hPD-L1 positive cases using the Mann-Whitney $\mathrm{U}$ test. The data are shown using Tukey box plots. Values of $\mathrm{P}<0.05$ were considered statistically significant. The actual P-values are shown in the graphs. 
Figure 7
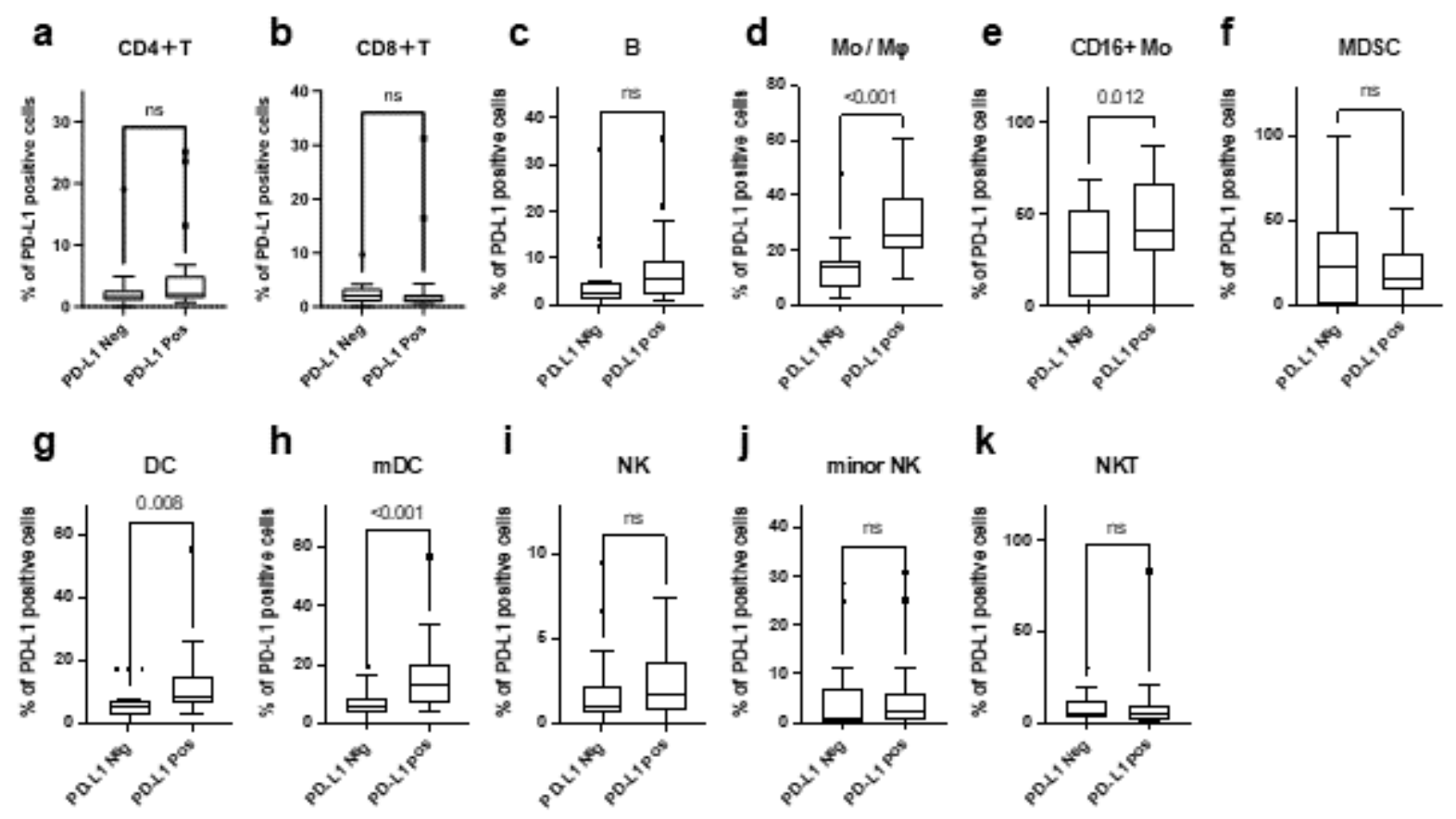

\section{Figure 7}

hPD-L1 positivity was associated with PD-L1 positive ratio in part of immune cell fraction. (a-k) For cases with tumor tissue samples, the percentages of PD-L1 positive cells in each immune cell fraction of the tumor tissues were compared between the hPD-L1-negative cases and hPD-L1 positive cases using the Mann-Whitney $\mathrm{U}$ test. The data are shown using Tukey box plots. The values of $\mathrm{P}<0.05$ were considered statistically significant. The actual P-values are shown in the graphs.

\section{Supplementary Files}

This is a list of supplementary files associated with this preprint. Click to download.

- FigureS1.pptx

- FigureS2.pptx

- FigureS3.pptx

- FigureS4.pptx

- FigureS5.pptx

- FigureS6.pptx

- Tables1.pptx

- Tables2.pptx

- Tables3.pptx 
- Tables4.pptx

- Tables5.pptx

- TableS6.pptx

- TableS7.pptx 